Research Paper

\title{
Rab14 Suppression Mediated by MiR-320a Inhibits Cell Proliferation, Migration and Invasion in Breast Cancer
}

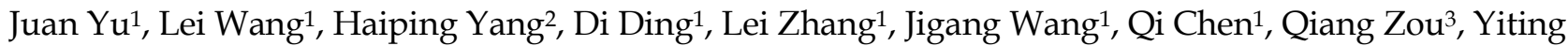 \\ $\mathrm{Jin}^{3}$, and Xiuping Liu ${ }^{4}$ \\ 1. Department of Pathology, School of Basic Medical Sciences, Fudan University, Shanghai 200032, China \\ 2. Department of Pathology, People's Hospital, Linzi District, Zibo City, Shandong 255400, China \\ 3. Department of Surgery, Huashan Hospital, Fudan University, Shanghai 200040, China \\ 4. Department of Pathology, the Fifth People's Hospital, Fudan University, Shanghai 200240, China \\ $\triangle$ Corresponding author: XiuPing Liu. Department of Pathology, the Fifth People's Hospital, Fudan University, Shanghai 200240, China 801 He Qing Road, \\ Shanghai, 200240, China. E-mail: xpliu1228@fudan.edu.cn.
}

() Ivyspring International Publisher. Reproduction is permitted for personal, noncommercial use, provided that the article is in whole, unmodified, and properly cited. See http://ivyspring.com/terms for terms and conditions.

Received: 2016.04.04; Accepted: 2016.09.17; Published: 2016.11.25

\begin{abstract}
We found that microRNA-320a (miR-320a) was an attractive prognostic biomarker in breast cancer (BC) previously, whereas its regulatory mechanism in BC was not well understood. Our aim was to identify miR-320a target gene, examine the clinical relationship between miR-320a and its target, and further explore the functions of its target in BC. In this study, miR-320a downstream target gene was determined in HEK-293T cells by dual luciferase reporter assay. Then western blotting and immunohistochemistry were used to assess miR-320a target gene expression in fresh frozen $(n=19$, breast cancer and matched non-malignant adjacent tissue samples) and formalin-fixed paraffin-embedded (FFPE) ( $n=130$, invasive BC tissues, the same panel detected for miR-320a expression previously) breast tissues, respectively. The results suggested that miR-320a could significantly suppressed Rab14 3'-untranslated region luciferase-reporter activity, and thus Rab14 was first identified as miR-320a target in BC. In 19 matched breast tissues, 12 (63\%) breast cancer tissues showed high expression of Rab 14 compared with the corresponding normal tissues. Rab14 immunoreactivity was mainly detected in the cytoplasm, $77 / 130(59.2 \%)$ showed high expression. Furthermore, Rab14 expression was found to be inversely correlated with miR-320a expression in fresh-frozen breast tissues as well as in FFPE invasive breast cancer samples. In addition, Rab14 expression levels were positively related to tumor size $(P=0.034)$, lymph node metastasis $(P<0.001)$, distant metastasis $(P=0.001)$, histological grade $(P=0.035)$ and clinical tumor lymph-node metastasis stage $(P=0.001)$. Patients with higher Rabl4 expression showed shorter overall survival time. Moreover, silencing of Rab14 could suppress proliferation, migration and invasion in breast cancer cell lines. Collectively, our results indicate that miR-320a could target Rab14 and that they could interact biologically in BC.
\end{abstract}

Key words: Rab14, miR-320a, breast cancer, proliferation, migration, invasion.

\section{Introduction}

Breast cancer (BC) remains one of the most common malignancies in women [1]. Despite the remarkable achievements we have made, the prognosis of patients with relapse or metastases is still disappointing. The molecular mechanisms underlying the progression of $\mathrm{BC}$ need to be further elucidated. Recently, mounting evidence indicates that miRNAs are dysregulated in BC and involved in breast tumorigenesis and metastasis. microRNAs (miRNAs) are small non-coding single-stranded RNAs and constitute a large class of gene regulators [2]. They exert their regulatory effects by binding to 3'-UTR of their mRNA targets, and thus repress target gene expression post-transcriptionally. It is estimated that $\geq 30 \%$ of human genes are regulated by miRNAs [3]. Therefore, it is not surprising that miRNAs can 
affect diverse cellular processes including cell proliferation, apoptosis, migration and invasion [4-7]. Many miRNAs are implicated in tumorigenesis. For example, miR-18a knockdown significantly decreases tumor growth by targeting STK4 in prostate cancer [8]. Ectopic expression of miR-372 inhibits cancer cell proliferation and invasion in hepatocellular carcinoma [9]. Overexpression of miR-10b in non-metastatic breast tumors initiates robust invasion and metastasis [10]. However, to date, no miRNAs have been utilized completely in clinical management, and further recognition of miRNAs may help to unravel their pathogenic role.

miR-320a is located at $8 \mathrm{p} 21.3$. Alteration of miR-320a expression has been implicated in multiple cancers, including hepatocellular, gastric and prostate cancers [11-13]. miR-320a could suppress colon cancer cell invasion by targeting $\beta$-catenin [14], where in oral cancer, miR-320a regulates angiogenesis by silencing neuropilin 1 [15]. In our previous study, miR-320a could serve as a potential biomarker for the prognosis of invasive $B C$, and dysregulation of miR-320a might be involved in BC progression [16]. Nevertheless, the downstream target gene of miR-320a to exert its regulatory effects in $\mathrm{BC}$ remained to be elucidated.

Here Rab14 was first identified as a target gene directly regulated by miR-320a. Rab14, a member of the large Ras-associated binding (Rab) family of low molecular mass GTPases, is associated with membrane trafficking between the Golgi complex and endosomes [17]. In contrast to members of the Ras and Rho families, their roles in human malignancies are less known. Findings suggested that Rabla was overexpressed in tongue squamous cell carcinomas compared with normal tissues [18]. Rab27A conferred invasive and metastatic promotion in breast cancer, which holded the potential for preventing breast cancer invasion and metastasis [19]. Nevertheless, few studies have been conducted on Rab14 in cancer. Our results suggested that Rab14 was overexpressed in BC tissues compared to adjacent normal tissues. The expression level of Rab14 was positively related with histological grade, clinical stage, lymph-node (LN) metastasis and distant metastasis. Moreover, Rab14 silencing could inhibit cell proliferation and migration/invasion in BC.

\section{Methods}

\section{Human samples}

A total of 130 formalin-fixed paraffin-embedded (FFPE) tissues were obtained from Huashan Hospital affiliated with Fudan University; the clinicopathological data of the patients were summarized, which were investigated in our previously published paper [20]. Nineteen pairs of breast cancer and corresponding normal tissue samples were collected and frozen in liquid nitrogen immediately after surgery and stored at $-70^{\circ} \mathrm{C}$ till use. All participants provided written informed consent and ethical approval was obtained from the Clinical Research Ethics Committee, Fudan University.

\section{Cell lines}

Human breast cancer cell line, SK-BR-3, MDA-MB-231 (M-231), MCF-7, T-47D, and BT-549 cells were obtained from the cell bank of Shanghai Institute for Biological Sciences. All cell lines were maintained in recommended medium supplemented with 10\% fetal bovine serum (FBS) (Invitrogen, Carlsbad, USA). The non-tumorigenic human breast epithelial cell line MCF-10A was a generous gift from Dr. Zhou (Fudan University, Shanghai, China) and cultured in DMEM/F-12 supplemented with 5\% horse serum (Invitrogen, Carlsbad, USA), $10 \mu \mathrm{g} / \mathrm{ml}$ insulin (Sigma-Aldrich, St. Louis, USA), $20 \mathrm{ng} / \mathrm{ml}$ EGF (Invitrogen, Carlsbad, USA), $100 \mathrm{ng} / \mathrm{ml}$ cholera toxin (Sigma-Aldrich, St. Louis, USA), $0.5 \mu \mathrm{g} / \mathrm{ml}$ hydrocortisone (Sigma-Aldrich, St. Louis, USA) and antibiotics. The cells were grown at $37{ }^{\circ} \mathrm{C}$ in a humidified incubator containing $5 \%$ carbon dioxide.

\section{Transfection}

Transient transfection of cells was achieved with Lipofectamine 2000 reagent (Invitrogen, Carlsbad, USA) according to the manufacturer's protocol. Pre-miR-320a, pre-miR-negative control (pre-miR-NC), anti-miR-320a, anti-miR-NC (Invitrogen, Carlsbad, USA), siRab14, and scrambled siRNA (siNC) (RiboBio, Guangzhou, China) were all transfected at a final concentration of $40 \mathrm{nmol} / \mathrm{L}$. Cells were harvested $24 \mathrm{~h}$ post-transfection for RNA analysis or $48 \mathrm{~h}$ post-transfection for analyzing protein levels.

\section{Real-time quantitative PCR (RT-qPCR)}

Total RNA was isolated from cells or tissues by Trizol reagent (Invitrogen, Carlsbad, USA). RNA quantity and quality was assessed using the Nanodrop 2000. The expression of miR-320a was detected with TaqMan Real-Time PCR Assays (Applied Biosystems, Foster City, USA) and normalized to RNU6 expression. mRNA was quantified following the manufacturer's instructions (Takara, Japan), with GAPDH as an internal reference. Primers used here are as follows: GAPDH (Forward 5'-GACCCCTTCATTGACCTCAAC-3', Reverse 5'-CTTCTCCATGGTGGTGAAGA-3'); and Rab14 (Forward 5'-GACAGATGCAAGGAATCTCACC-3', Reverse (5'-GCTTCGAGGAACAATAAGCCAT-3'). 
All reactions were run in ABI Prism 7900 Sequence Detector System (Applied Biosystems, Foster City, USA). Relative quantification was determined by the $2^{-\triangle \Delta C T}$ method [21].

\section{Cell proliferation}

Cell proliferation was determined by Cell Counting Kit- 8 assay (Dojindo, Japan) according to the protocol. Cells were plated in 96-well plates. Plates were measured at $450 \mathrm{~nm}$ by spectrophotometer (Thermo Fisher Scientific, Waltham, USA) after incubating 2 hours.

\section{Cell migration and invasion assays}

The migration and invasion assays were performed by Transwell insert chambers $(8-\mu \mathrm{m}$ pore size, Corning, USA). For transwell assay, $5 \times 10^{4}$ or $1 \times 10^{5}$ cells in serum-free media were placed into the upper chamber coated with or without Matrigel (BD Biosciences, San Jose, USA). Serum containing FBS was used as a chemo-attractant in the lower chamber. After several hours of incubation, the cells remaining in the upper chamber were removed by a cotton swab. The insert was fixed in methanol and stained with crystal violet. The cells were photographed and quantified by Vectra Automated Quantitative Pathology Imaging System (PerkinElmer, USA).

\section{Luciferase assay}

Pluc-Rab14-3'UTR-wt was obtained from RiboBio (Guangzhou, China) and the corresponding mutation constructs were achieved by Fast Mutagenesis System (Transgen Biotech, Beijing, China). The sequences for the primers are as follows: plucRab14-3'UTRwt (Forward 5'-ccgctcgagtgcctcattgt cttctgtacatc-3', Reverse 5'-gaatgcggccgctgactgcacaagc agtaagc-3'), plucRab14-3'UTRmu1 (Forward 5'-ctcgtgcttaattaattggtatatcttttttta-3', Reverse 5'taccaattaattaagcacgagagagaagata- $3^{\prime}$ ) and plucRab14-3 'UTRmu2 (Forward 5'-ctaagtaatagacccactggtatattcttgt gt-3', Reverse (5'- taccagtgggtctattacttagtgatgtggtt-3'). HEK-293T cells were cultured in 24-well plates, co-transfected with 100-ng pluc-3'-UTR and 10-nmol pre-miR-320a or pre-miR-NC; $24 \mathrm{~h}$ after transfection, luciferase activities were measured with a Dual-Luciferase Reporter System (Promega, Madison, USA) on a Glomax Luminometer (Promega, Madison, USA). Renilla luciferase activity was normalized to Firefly luciferase activity for each transfected well.

\section{Western blotting}

Cells were lysed with RIPA buffer [50 mM Tris, PH 8.0, $150 \mathrm{mM} \mathrm{NaCl}, 1 \%$ NP-40, 0.1\% SDS, 0.5\% sodium deoxycholate, $2 \mathrm{mM}$ EDTA, $0.02 \% \mathrm{NaN} 3$ ] with protease inhibitor Cocktail (Roche Applied Science, Indianapolis, USA). Lysates were SDS-PAGE gel electrophoresis followed by electrophoretic transfer to a polyvinylidenedifluoride membrane (Millipore, Bedford, USA) and immunoblotting with the indicated antibodies. Antibodies used were as follows: anti-Rab14 (1:2000; polyclonal rabbit anti-human, Cat.\#ab40938, Abcam, USA) and anti- $\beta$-actin (1:2000; monoclonal mouse anti-human, Cat.\#a5441, Sigma-Aldrich, St. Louis, USA). Bound antibodies were visualized by chemiluminescence (West Pico Super Signal; Pierce, USA). Experimental details was described previously [22].

\section{Immunohistochemistry}

Slides were deparaffinized in xylene and rehydrated in graded alcohols. Antigen retrieval was performed with $0.01 \mathrm{M}$ citrate buffer at $\mathrm{pH} 6.0$ at $95^{\circ} \mathrm{C}$ for $12 \mathrm{~min}$. Then, slides were incubated with primary antibody (anti-Rab14, 1:100) for $12 \mathrm{~h}$ at $4^{\circ} \mathrm{C}$, followed by incubation with secondary antibody for $1 \mathrm{~h}$ at room temperature. IHC staining was examined by two independent pathologists. The experimental detail was performed as previously. For statistical analysis, the percentage of positive tumor cells and staining intensity were evaluated over 10 visual fields at a power of $400 \times$ by a microscope (Carl Zeiss, Germany). The staining index (SI) was calculated as: $\mathrm{SI}=$ staining intensity $\mathrm{x}$ proportion of positively stained tumor cells, and it resulted in a score of 0 to 9 . Analysis in detail was performed as described previously [16]. Cutoff value of Rab14 was chosen by a measurement of heterogeneity with the log-rank test statistic with respect to overall survival, and a score of 4 was chosen [23, 24]. The expression levels of Rab14 were defined as high $(\mathrm{SI} \geq 4)$ or low $(\mathrm{SI}<4)$.

\section{Statistical analysis}

IBM SPSS 19 (SPSS, USA) was used for statistical analysis. Unless otherwise specified, student's t-test was applied to determine significant differences (two-tailed, $\quad P<0.05$ ). Spearman correlation coefficients were performed to determine the association between Rab14 and miR-320a expression. Kaplan-Meier survival method was performed to assess the prognostic significance of Rab14, and the survival difference was analyzed by log-rank test. All values in the study were expressed as the mean \pm SEM, a $P<0.05$ was defined as statistically significant.

\section{Results}

\section{Rabl4 is a target of miR-320a in breast cancer cell lines}

We previously reported that down-regulation of miR-320a was inversely associated with the progression and prognosis of invasive $\mathrm{BC}$, this study was initiated to investigate the potential mRNA 
targets downstream of miR-320a in BC. To this end, we used target gene prediction algorithm TargetScan (http://www.targetscan.org/) to search for miR-320a targets. Among the predicted targets, Rab14 was of particular interest. We found that Rab14 3'-UTR contained two putative binding sites for miR-320a at nucleotide position 597-603 and 2262-2269. To further verify the direct effect of miR-320a on Rab14, wild-type and mutant Rab14 3'-UTR containing miR-320a binding sites were cloned into the downstream of the firefly luciferase reporter gene in the Pluc vector (Figure 1A). We next proceeded to conduct a reporter assay in HEK-293T cells by co-transfecting pluc-Rab14-wt-3'UTR (wt-3'UTR) or its mutant (mut-3'UTR) with pre-mir-320a or pre-miR-NC. Indeed, as shown in Figure 1B, miR-320a sharply decreased the relative luciferase activity of the wt-3'UTR of Rab14, but not of mut-3'UTR, in which two putative binding sites were jointly mutated.

We assessed Rab14 expression in a series of breast epithelial cell lines, as shown, Rab14 protein level was higher in breast cancer cell lines compared with MCF-10A (Figure 1C). We also examined miR-320a expression in the same cell lines detected for Rab14 expression, miR-320a expression level was found to be significantly lower in breast cancer cells, when compared with human mammary epithelial cell MCF-10A (data shown elsewhere).

A

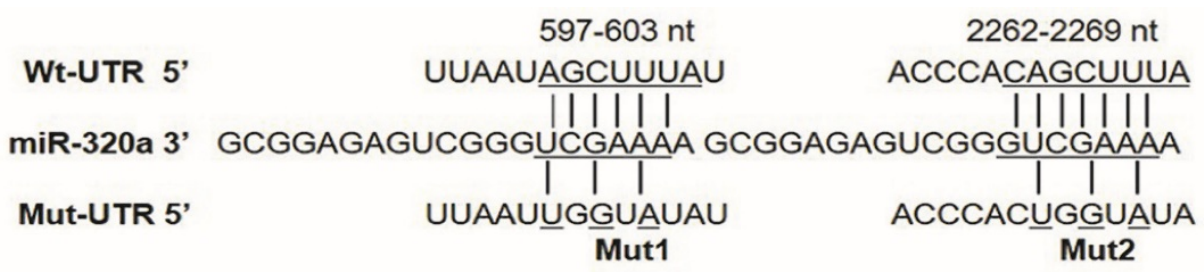

B

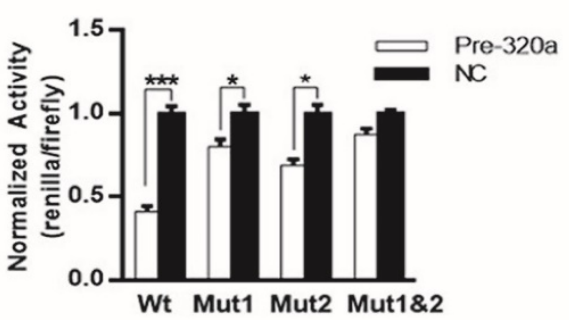

C

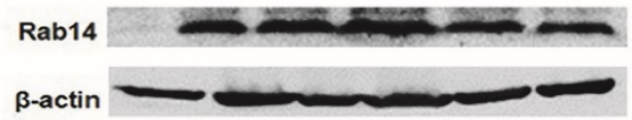

MCF10A SK-BR-3 T47D MCF-7 BT-549 M-231

D

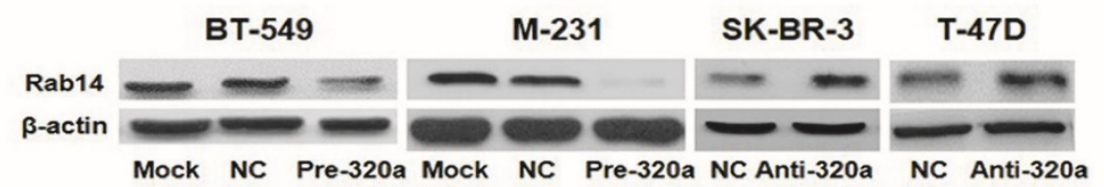

E
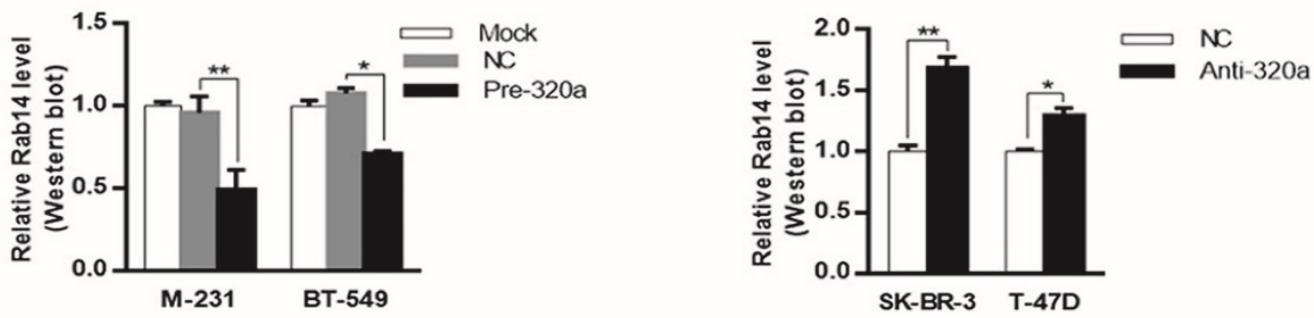

$\mathbf{F}$
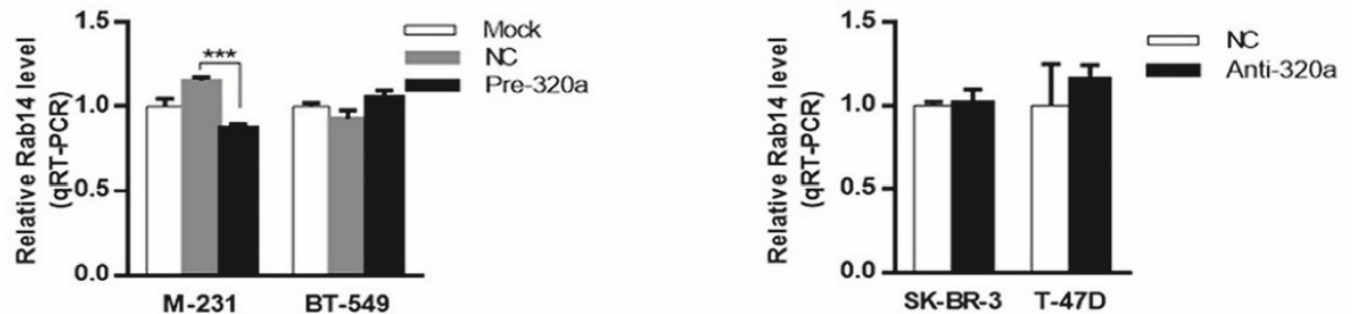

Figure 1 Rabl4 is a target of miR-320a. a, Schematic of the two predicted binding sequences between miR-320a and Rabl4 3’UTR at nucleotide 597-603 and 2262-2269, the pluc-Rab14-3'UTR-reporter constructs containing the wild-type (wt) or mutated (mut) sequences (underlined). b, Luciferase assay in HEK-293T cells. miR-320a suppressed the activity of Rab 14-3'UTR-wt, whereas the joint mutation sequences (mut1\&2) abolished the suppression in HEK-293T cells. c, Rab14 expression level by western blot in a series of breast epithelial cell lines. d, Rab14 protein level measured by western blot after transfection with pre-mir-320a or anti-miR-320a for 48 hours, respectively. e, Histogram figure about the densitometric analysis of Rab14 protein expression. f, RT-qPCR for Rab14 expression after transfection with pre-miR-320a or anti-miR-320a for 24 hours, GAPDH was used as an endogenous control. All experiments were repeated three times. $* P<0.05$, $* * P<0.01$. 
Moreover, western blot analysis (Figure 1D and E) demonstrated that miR-320a significantly suppressed the protein level of Rab14 in both MDA-MB-231 and BT-549 breast cancer cell lines by transfection of miR-320a precursor. In addition, RT-qPCR analyses revealed that miR-320a elicited degradation of Rab14 mRNA in MDA-MB-231 cell line, but it did target Rab14 through translational inhibition in BT-549 cell (Figure 1F). In support of this, we silenced miR-320a expression by transfection of anti-miR-320a into SK-BR-3 and T-47D cells, and we observed a clear induction in the protein level of Rab14 in anti-miR-320a-transfected SK-BR-3 and T-47D cells, whereas the levels of Rab14 mRNA showed no difference. Taken together, Rab14 was a downstream target gene of miR-320a.

\section{Rab14 expression is negatively associated with the expression of $\mathrm{miR}-320 \mathrm{a}$ in fresh frozen and FFPE breast tissues}

To analyze miR-320a-Rab14 interactions in human BC, we detected the expression of miR-320a and Rab14 in 19 paired BC and adjacent normal tissues using RT-qPCR and western blot, respectively. As expected, almost all tumor samples showed significant downregulation of miR-320a expression with respect to the adjacent non-tumor tissues (Wilcoxon signed-rank test, $P<0.001$, Figure 2A). Nevertheless, 12 of 19 cancer tissues $(63 \%)$ showed high Rab14 expression and 4 tumors (21\%) exhibited low expression compared with paired normal tissues (Figure 2B). Patients with high Rab14 expression tended to show low miR-320a expression in fresh frozen BC tissues (Spearman's $r=-0.69, P<0.01$, Figure 2C).
A

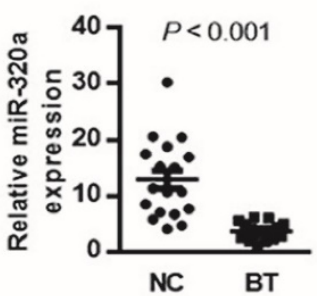

B

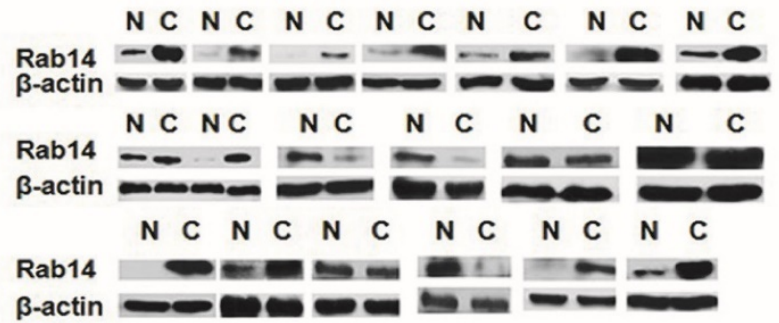

C

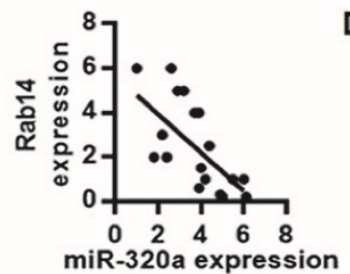

$\mathbf{E}$

\begin{tabular}{ccccc}
\hline miR-320a & \multicolumn{4}{l}{ Rab14 expression } \\
expression & High & Low & Total & $\boldsymbol{P}$ \\
\hline High & 18 & 42 & 60 & $<0.001$ \\
Low & 59 & 11 & 70 & \\
Total & 77 & 53 & 130 & \\
\hline
\end{tabular}

G

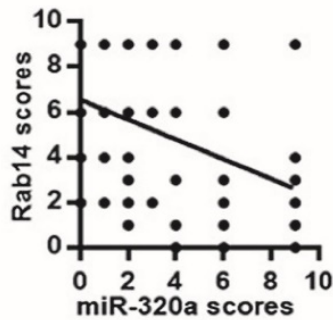

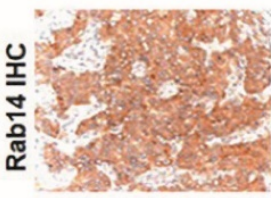

Score 3

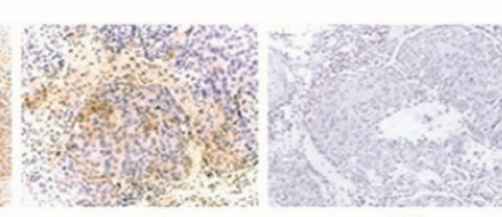

Score 1

NC

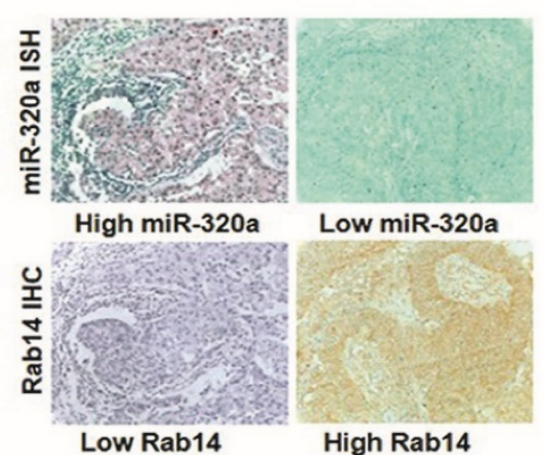

Figure 2. Rab14 and miR-320a might interact biologically. a, miR-320a expression in 19 paired fresh breast tissues by RT-qPCR. b, Rab 14 expression in 19 sets of fresh BC and the adjacent non-tumor tissues assessed by western blot. c, Spearman correlation analysis between miR-320a and Rabl4 in fresh frozen breast tissues $(r=-0.69, P<0.01)$. $d$, Representative case of Rab14 staining intensity. e, Rab14 and miR-320a expression level in 130 FFPE invasive BC tissues. Rab14 was immunostained with anti-Rab14 antibody, and the consecutive sections were detected for miR-320a with locked nucleic acid probes. f, Representative image of Rab-14 and miR-320a expression. g, Spearman correlation analysis between miR-320a and Rab14 in $130 \mathrm{FFPE}$ invasive BC samples $(r=-0.48, P<0.001)$. Images were taken with a power of $\times 200$. 
To further evaluate Rab14 expression in human BC, IHC was performed in 130 invasive BC tissues. Rab14 immunoreactivity was mainly detected in the cytoplasm (Figure 2D), 77/130 (59.2\%) showed high Rab14 expression (SI $\geq 4)$ and the remaining 53/130 (40.8\%) revealed low expression of Rab14 (Figure 2E). Given our previous study of miR-320a by in situ hybridization (ISH), together with Rab14 IHC staining on consecutive tissue sections, patients who exhibited stronger Rab14 expression tended to show down-regulated miR-320a levels in BC tissue samples, and vice versa (Figure 2F). An inverse association between miR-320a and Rab14 was identified (Spearman's $r=-0.48, P<0.001$, Figure 2G), which corresponded with a similar expression pattern in fresh-frozen breast cancer tissues as compared with adjacent normal tissues, suggested that increased Rab14 expression might result from miR-320a down-regulation in human BC. Collectively, Rab14 and miR-320a might interact biologically in BC.

\section{Rab14 expression in relation to clinicopathological characteristics}

We also analyzed Rab14 expression and its association with clinicopathological factors. As shown in Table 1, Rab14 expression was not linked to age, estrogen receptor (ER), progesterone receptor (PR), Her-2 expression or menopause status, but it was significantly positively related to tumor size $(P=$ $0.034)$, LN metastasis $(P<0.001)$, distant metastasis $(P$ $=0.001)$, histological grade $(P=0.035)$ and clinical TNM stage $(P=0.001)$. The results revealed that Rab14 was overexpressed in high grade BC tissues. Accordingly, we assessed the prognostic value of Rab14 expression in selective patient subgroups stratified by LN status and clinical stage. As shown in Figure $3 \mathrm{~A}$, patients with $\mathrm{LN}$ metastasis exhibited a significant difference in overall survival $(P=0.044)$, whereas no significant correlation for the overall survival in the subgroups, including LN negative and different clinical stage patients, were found. In addition, among the 130 invasive BC patients, the overall survival rate of patients with high Rab14 expression tended to be lower than that of patients with low expression of $\operatorname{Rab} 14$ ( $P=0.001$, Figure 3B). Our results suggested that Rab14 overexpression was associated with a poor prognosis of BC.

\section{Rab 14 plays a key role on proliferation, migration and invasion in human breast cancer cell lines.}

To explore the biological roles of Rab14, we first examined the expression of Rab14 by transfection of a specific small interfering RNA against Rab14 into MDA-MB-231 and BT-549 cells. As shown in Figure
4A, B and C, siRab14 transfection led to decreased Rab14 expression both in protein and mRNA level. Furthermore, in the MDA-MB-231cell line, silencing of Rab14 significantly decreased the viability by $36 \%$ at the fourth day; whereas siRab14 did not reduce the viability of BT-549 cells (Figure 4D). Then, we asked whether Rab14 could affect cell migration and invasion, indeed, using transwell assay, siRab14 did reduce migration for both MDA-MB-231 and BT-549 cells, and Rab14 depletion significantly reduced cell invasiveness (Figure 4E). These data indicated that Rab14 inhibition could impair the proliferation, migration and invasion of breast cancer cells.

Table 1. Correlation of Rab14 expression and the clinicopathological characteristics in invasive BC $(n=130)$.

\begin{tabular}{|c|c|c|c|c|}
\hline Characteristics & $\mathrm{n}$ & $\begin{array}{l}\text { Rab14 e } \\
\text { High ex } \\
\text { Low exp }\end{array}$ & $\begin{array}{l}\text { on } \\
n, n(\%) \\
n, n(\%)\end{array}$ & $P$ value \\
\hline Age (years) & & & & 0.574 \\
\hline$<45$ & 15 & $7(47)$ & $8(53)$ & \\
\hline $45-55$ & 61 & $37(61)$ & $24(39)$ & \\
\hline$>55$ & 54 & $33(61)$ & $21(39)$ & \\
\hline Tumor size $(\mathrm{cm})$ & & & & 0.034 \\
\hline$\leq 2.5$ & 79 & $41(52)$ & $38(48)$ & \\
\hline$>2.5$ & 51 & $36(71)$ & $15(29)$ & \\
\hline Lymph node metastasis & & & & $<0.001$ \\
\hline 0 & 71 & $30(42)$ & $41(58)$ & \\
\hline $1-2$ & 32 & $24(75)$ & $8(25)$ & \\
\hline$>2$ & 27 & $23(85)$ & $4(15)$ & \\
\hline Distant metastasis & & & & 0.001 \\
\hline Yes & 118 & $65(55)$ & $53(45)$ & \\
\hline No & 12 & $12(100)$ & $0(0)$ & \\
\hline Histological grade & & & & 0.035 \\
\hline well (I) & 14 & $5(36)$ & $9(64)$ & \\
\hline moderate (II) & 93 & $54(58)$ & $39(42)$ & \\
\hline poor (III) & 23 & $18(78)$ & $5(22)$ & \\
\hline Clinical TNM stage & & & & 0.001 \\
\hline I & 44 & $18(41)$ & $26(59)$ & \\
\hline II & 54 & $32(59)$ & $22(41)$ & \\
\hline III-IV & 32 & $27(84)$ & $5(16)$ & \\
\hline Estrogen receptor & & & & 0.756 \\
\hline- & 61 & $37(61)$ & $24(39)$ & \\
\hline+ & 69 & $40(58)$ & $29(42)$ & \\
\hline Progesterone receptor & & & & 0.835 \\
\hline- & 75 & $45(60)$ & $30(40)$ & \\
\hline+ & 55 & $32(58)$ & $23(42)$ & \\
\hline Her-2 expression & & & & 0.888 \\
\hline- & 50 & $30(60)$ & $20(40)$ & \\
\hline+ & 80 & $47(59)$ & $33(41)$ & \\
\hline Menopause & & & & 0.553 \\
\hline No & 50 & $28(56)$ & $22(44)$ & \\
\hline Yes & 80 & $49(61)$ & $31(39)$ & \\
\hline
\end{tabular}

\section{Discussion}

Given that the biological effects of altered miRNA expression rely on the significance of their target genes, miRNAs could have various effects in different tissues depending on the biological role of their targets in the specific tissue. One report 
demonstrated that miR-320a could inhibit leukemic cell proliferation by targeting transferring receptor 1 (CD71) [25]. Another study indicated that miR-320a could inhibit tumor invasion by targeting neuropilin [26]. We previously reported that miR-320a was down-regulated in invasive BC compared with carcinoma in situ. Until recently, very few studies have been performed to identify the mRNA effector of miR-320a in BC. Here Rab14 was first identified as a direct target gene of miR-320a in breast cancer cell lines. Analyses of multiple breast cancer cell lines, fresh-frozen BC tissues and FFPE invasive BC samples showed that decreased miR-320a expression paralleled an increase in Rab14 protein levels, which suggested that downregulation of miR-320a was to be an event responsible for Rab14 overexpression in BC. Identification of Rab14 modulated by miR-320a was helpful to clarify complicated mechanisms in BC development.
A

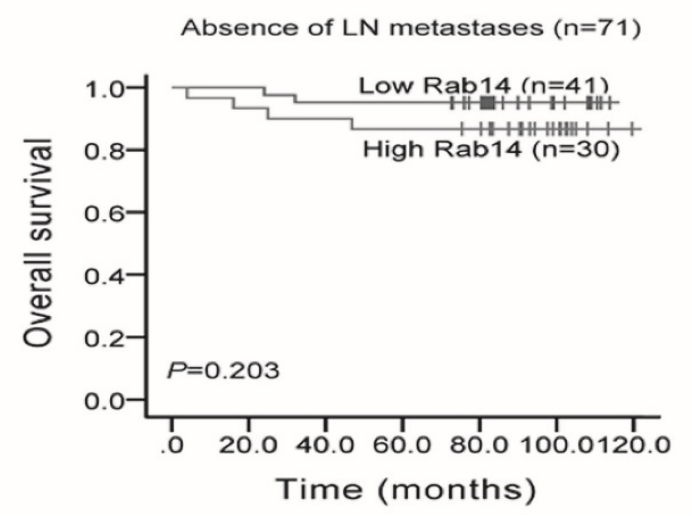

TNM I-II $(n=98)$

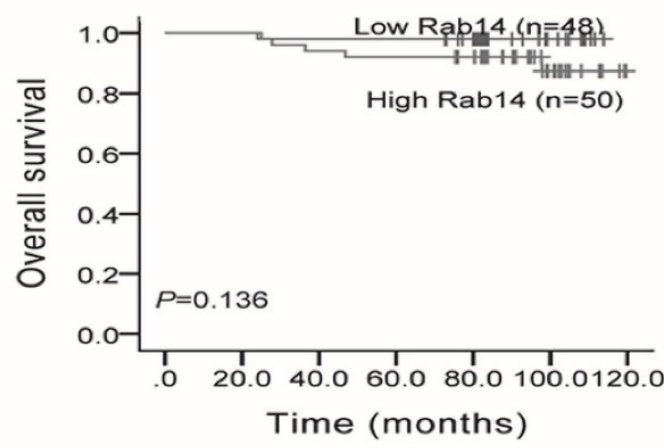

B

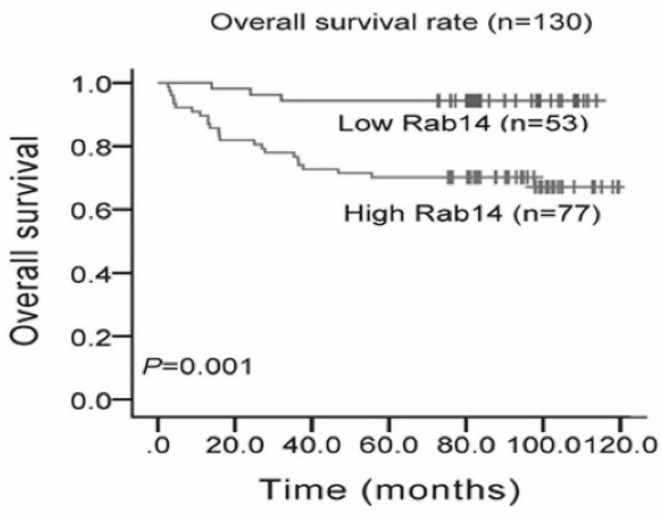

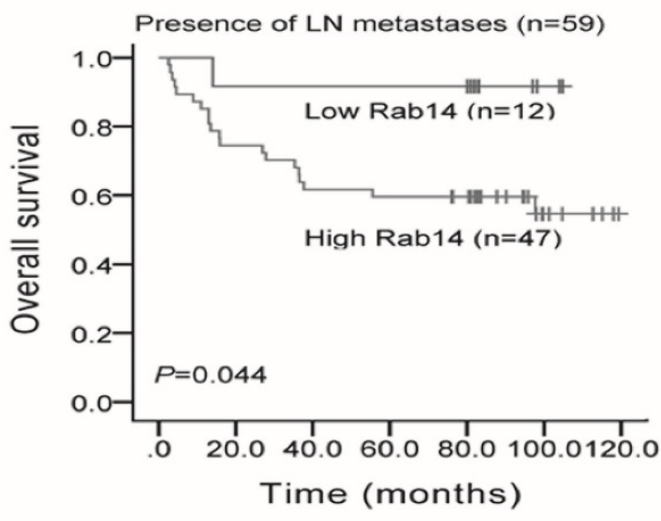

TNM III-IV $(n=32)$

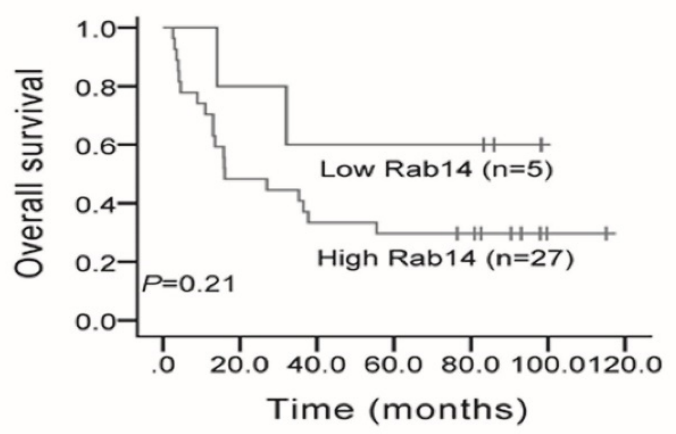

to clinical stage. b, Overall survival rate of all patients with high or low Rab14 expression. 
A

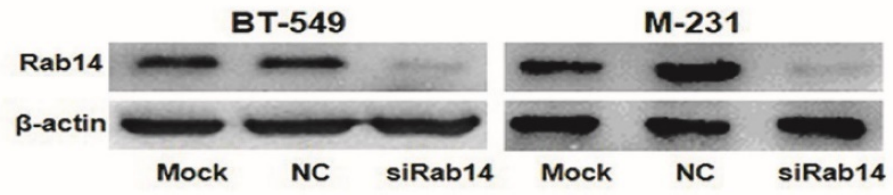

B

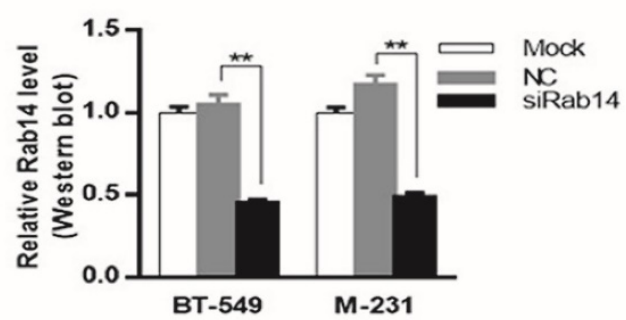

C

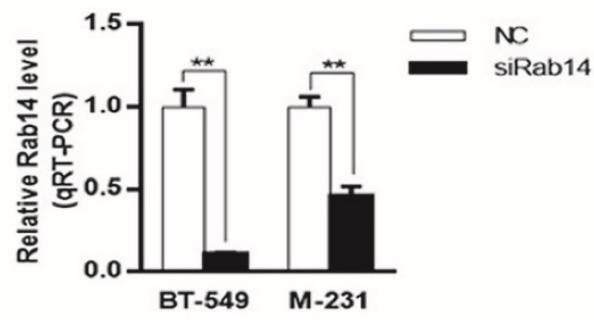

D
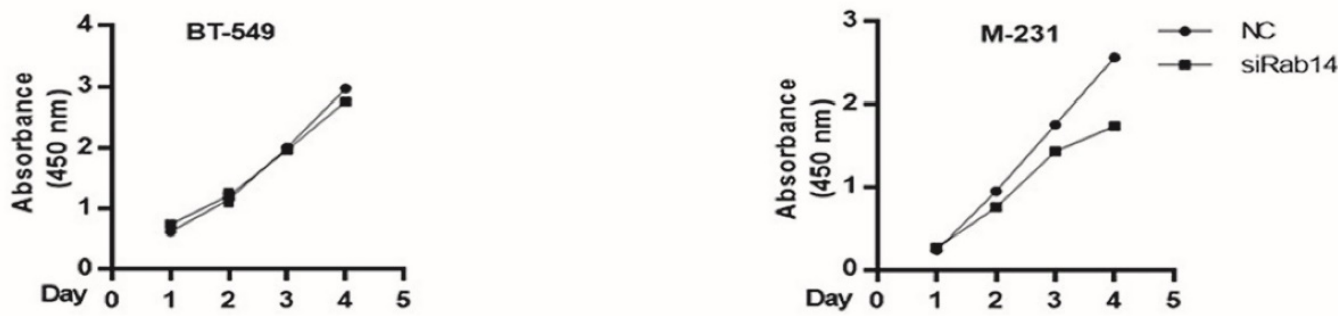

E
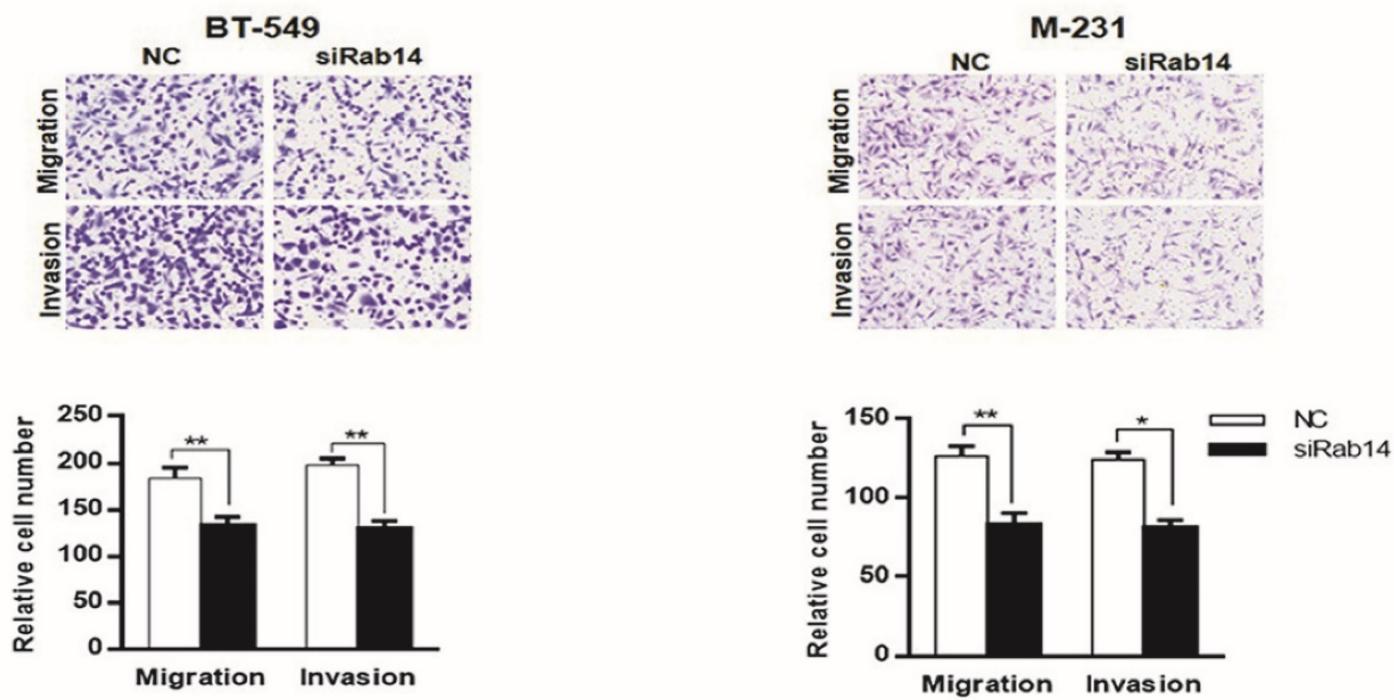

Figure 4 knockdown of Rab14 led to reduced viability, migration and invasion in breast cancer cells. a, Validation of Rab14 suppression by siRab14 using western blot. b, histogram related to densitometric analysis of Rabl4 protein expression level. c, RT-qPCR for Rabl4 expression after transfection of siRabl4 for 24 hours. d, Effects of siRabl4 on the proliferation of MDA-MB-231 and BT-549 cells. e, Transwell cell migration and invasion assay of MDA-MB-231 and BT-549 cells transfected with siRab14 or negative control. Cells were counted microscopically $(\times 200)$. All experiments were repeated three times. $* P<0.05, * * P<0.01$.

Rab14 is a member of the Rab protein family, which participated in Rab-mediated vesicle budding, uncoating, docking and fusion [27]. It has emerged as a major regulator of intracellular membrane trafficking [28], and studies have shown that Rab14 play a role in cell migration events by regulating the turnover of $\mathrm{N}$-cadherin at the cell surface [29]. However, few studies have been performed to identify the roles of Rab14 in cancer. One paper reported that Rab14 was identified as a lung cancer-specific protein, and recent evidence suggested that Rab14 was up-regulated in non-small cell lung cancer (NSCLC) compared with corresponding non-tumor lung tissues [30, 31]. In our study, Rab14 was overexpressed in fresh-frozen breast cancer samples compared with adjacent normal tissues using western blotting. Moreover, for the first time, Rab14 expression was evaluated in FFPE invasive BC tissues and its relationship with the clinicopathological features of BC was assessed. IHC staining for Rab14 was performed. Rab14 was mainly detected in the cytoplasm. High expression of Rab14 was significantly positively associated with increased malignancy of BC. In line with this, Kaplan-Meier 
survival analysis showed that high Rab14 expression exhibited significant associations with poor prognosis. Taken together, Rab14 might serve as an indicator for the prognosis of BC. Nevertheless, no significant relations of Rab14 expression for patients subgrouped by clinical stage were found which might be due to the small subgroup sample size.

These results suggested potential oncogenic functions of Rab14 in BC, and then we sought to determine the role of Rab14. Knockdown of Rab14 could significantly inhibit proliferation in MDA-MB-231 cells; the same effects were not observed in BT-549 cells, which may be attributed to various cellular contexts. Reduction of Rab14 led to the inhibition of cell migration and invasion in vitro. Rab14 is conserved among mammals, and its importance in tumors may not be limited to BC. Further investigation of Rab14 is warranted to better clarify its role. Recent evidence has shown that the up-regulation of Rab14 is partially associated with nasopharyngeal carcinoma radio resistance [32].

\section{Conclusion}

In conclusion, this study indicates that miR-320a could negatively regulate Rab14. Our findings also highlight the role of Rab14 in BC, which could provide insight into the basic mechanisms of breast cancer initiation and progression.

\section{Abbreviations}

BC: breast cancer; microRNAs: miRNAs; microRNA-320a: miR-320a; 3'UTR: 3'-untranslated region; FFPE: formalin-fixed paraffin-embedded; IHC: immunohistochemistry; ISH: in situ hybridization; LN: lymph node; NC: negative control; RT-qPCR: quantitative real time PCR; TNM: tumor lymph-node metastasis; wt: wild type; mut: mutant; siRNA: small interfering RNA; SI: staining index; Rab: Ras-associated binding; ER: estrogen receptor; PR: progestin receptor.

\section{Acknowledgements}

This work was supported by grants from the National Nature Science Foundation of China (No. 81272387 and No. 81470857) and Chun-Tsung Endowment (No. 11019, to L. Wang). We thank members of our laboratory for helpful discussions. The paper was edited by a native English speaker at AJE (American journal experts, www.aje.com/certificate). The Certificate Verification Key is 5B85-EEDB-701C-B00A-698C.

\section{Ethical Statement}

All participants provided written informed consent at the time of initial diagnosis and ethical approval was obtained from the Clinical Research Ethics Committee, Fudan University.

\section{Competing interests}

The authors declare that they have no competing interests.

\section{References}

1. Siegel R., et al. Cancer statistics, 2014. CA: A Cancer Journal for Clinicians, 2014. 64(1): 9-29.

2. Krol J., et al. The widespread regulation of microRNA biogenesis, function and decay. Nat Rev Genet, 2010. 11(9): 597-610

3. Lewis B.P., et al. Conserved Seed Pairing, Often Flanked by Adenosines, Indicates that Thousands of Human Genes are MicroRNA Targets. Cell. 2005;120(1): 15-20.

4. Palma C.A., et al. MicroRNA-155 as an inducer of apoptosis and cell differentiation in Acute Myeloid Leukaemia. Mol Cancer, 2014. 13: 79.

5. Fang Y., et al. MicroRNA-7 inhibits tumor growth and metastasis by targeting the phosphoinositide 3-kinase/Akt pathway in hepatocellular carcinoma. Hepatology, 2012. 55(6): 1852-62.

6. Bockhorn J., et al. MicroRNA-30c targets cytoskeleton genes involved in breast cancer cell invasion. Breast Cancer Res Treat, 2013. 137(2): 373-82.

7. Liu J., et al. MicroRNA-144 inhibits the metastasis of gastric cancer by targeting MET expression. J Exp Clin Cancer Res, 2015. 34(1): 35.

8. Hsu T.I., et al. MicroRNA-18a is elevated in prostate cancer and promotes tumorigenesis through suppressing STK4 in vitro and in vivo. Oncogenesis, 2014. 3: e99.

9. Wu G., et al. Low mir-372 expression correlates with poor prognosis and tumor metastasis in hepatocellular carcinoma. BMC Cancer, 2015. 15: 182.

10. Ma L., et al. Weinberg, Tumour invasion and metastasis initiated by microRNA-10b in breast cancer. Nature, 2007. 449(7163): 682-8.

11. Ishida H., et al. Alterations in microRNA expression profile in $\mathrm{HCV}$-infected hepatoma cells: involvement of miR-491 in regulation of HCV replication via the PI3 kinase/Akt pathway. Biochem Biophys Res Commun, 2011. 412(1): 92-7.

12. Xu Q., et al. Expression of serum miR-20a-5p, let-7a, and miR-320a and their correlations with pepsinogen in atrophic gastritis and gastric cancer: a case-control study. BMC Clin Pathol, 2013. 13: 11

13. Hsieh I.S., et al. MicroRNA-320 suppresses the stem cell-like characteristics of prostate cancer cells by downregulating the Wnt/beta-catenin signaling pathway. Carcinogenesis, 2013. 34(3): 530-8.

14. Sun J.Y., et al. MicroRNA-320a suppresses human colon cancer cell proliferation by directly targeting beta-catenin. Biochem Biophys Res Commun, 2012. 420(4): 787-92.

15. Wu Y.Y, et al. miR-320 regulates tumor angiogenesis driven by vascular endothelial cells in oral cancer by silencing neuropilin 1. Angiogenesis, 2014. 17(1): 247-60.

16. Yang H., et al. miR-320a is an independent prognostic biomarker for invasive breast cancer. Oncol Lett, 2014. 8(3): 1043-1050.

17. Junutula J.R., et al. Rab14 is involved in membrane trafficking between the Golgi complex and endosomes. Mol Biol Cell, 2004. 15(5): 2218-29.

18. Shimada K., et al. Aberrant expression of RAB1A in human tongue cancer. Br J Cancer, 2005. 92(10): 1915-21.

19. Wang J.S., et al. Enhanced expression of Rab27A gene by breast cancer cells promoting invasiveness and the metastasis potential by secretion of insulin-like growth factor-II. Mol Cancer Res, 2008. 6(3): 372-82.

20. Yang H.P., et al. miR-320a is an independent prognostic biomarker for invasive breast cancer. Oncology Letters, 2014. 8(3): 1043-1050.

21. Livak K.J and Schmittgen T.D. Analysis of relative gene expression data using real-time quantitative PCR and the 2(-Delta Delta $\mathrm{C}(\mathrm{T})$ ) Method. Methods, 2001. 25(4): 402-8.

22. Cao X.X., et al. RACK1 promotes breast carcinoma proliferation and invasion/metastasis in vitro and in vivo. Breast Cancer Res Treat, 2010. 123(2): 375-86.

23. Li W., et al. Sphingosine kinase 1 is associated with gastric cancer progression and poor survival of patients. Clin Cancer Res, 2009. 15(4): 1393-9.

24. Li X., et al. Transducin (beta)-like 1 X-linked receptor 1 promotes proliferation and tumorigenicity in human breast cancer via activation of beta-catenin signaling. Breast Cancer Res, 2014. 16(5): 465.

25. Schaar D.G., et al. miR-320 targets transferrin receptor 1 (CD71) and inhibits cell proliferation. Exp Hematol, 2009. 37(2): 245-55.

26. Zhang Y., et al. microRNA-320a inhibits tumor invasion by targeting neuropilin 1 and is associated with liver metastasis in colorectal cancer. Oncol Rep, 2012. 27(3): 685-94.

27. Park S.Y., et al. Crystal structures of human TBC1D1 and TBC1D4 (AS160) RabGTPase-activating protein (RabGAP) domains reveal critical elements for GLUT4 translocation. J Biol Chem, 2011. 286(20): 18130-8.

28. Kelly E.E., et al. Class I Rab11-family interacting proteins are binding targets for the Rab14 GTPase. Biol Cell, 2010. 102(1): 51-62. 
29. Linford A., et al. Rab14 and its exchange factor FAM116 link endocytic recycling and adherens junction stability in migrating cells. Dev Cell, 2012. 22(5): 952-66.

30. Wang R., et al. MicroRNA-451 functions as a tumor suppressor in human non-small cell lung cancer by targeting ras-related protein 14 (RAB14). Oncogene, 2011. 30(23): 2644-58.

31. Zhang HZ, et al. Analysis and identification of tumor marker in lung cancer using two-dimensional gel electrophoresis and matrix-assisted laser desorption ionization time of flight mass spectrometry. Life Science, 2009; 3: 46-53.

32. Zhang T., et al. MiR-451 increases radiosensitivity of nasopharyngeal carcinoma cells by targeting ras-related protein 14 (RAB14). Tumour Biol, 2014. 35(12): 12593-9. 